\title{
SEEDING EXPERIMENTS AT SPARC
}

\author{
Marie Labat, Franco Ciocci, Giuseppe Dattoli, Mario Del Franco, Andrea Doria, \\ Gian Piero Gallerano, Luca Giannessi, Emilio Giovenale, Alberto Maria Antonio Petralia, \\ Marcello Quattromini, Concetta Ronsivalle, Elio Sabia, Ivan Spassovsky, Vincenzo Surrenti \\ ENEA C.R. Frascati, Italy \\ Bertrand Carr, David Garzella, CEA, Gif-sur-Yvette, France \\ Alessandro Cianchi, Barbara Marchetti, INFN-Roma II, Roma, Italy \\ Mario Mattioli, Maurizio Serluca, INFN-Roma, Roma, Italy \\ David Alesini, Marco Bellaveglia, Roberto Boni, Manuela Boscolo, \\ Michele Castellano, Enrica Chiadroni, Alberto Clozza, Luca Cultrera, \\ Giampiero Di Pirro, Alessandro Drago, Massimo Ferrario, Luca Ficcadenti, \\ Daniele Filippetto, Valeria Fusco, Alessandro Gallo, Giancarlo Gatti, \\ Andrea Mostacci, Elisabetta Pace, Luigi Palumbo, Bruno Spataro, \\ Cristina Vaccarezza, INFN/LNF, Frascati, Italy \\ Alberto Bacci, Vittoria Petrillo, Andrea Renato Rossi, Luca Serafini \\ Istituto Nazionale di Fisica Nucleare, Milano, Italy \\ Fabien Briquez, Marie-Emmanuelle Couprie, SOLEIL, Gif-sur-Yvette, France \\ Gabriel Marcus, James Rosenzweig, UCLA, Los Angeles, California, USA
}

\begin{abstract}
The SPARC Free Electron Laser can be operated in both SASE and seeded modes. A major part of the second stage of the commissioning, now undergoing, is dedicated to the characterization of the SASE radiation. Simultaneously, we are finalizing the experimental setup for seeding. We present an in-situ characterization of the two input seeds that are foreseen: both are obtained via harmonic generation, the first one in crystal (400 and $266 \mathrm{~nm}$ ) and the second in rare gas (Argon). We also describe the specific diagnostics implemented for the electron-seed overlap in the undulator, together with the diagnostics for radiation analysis (2D spectrometer and FROG). The seeding will enable the operation of the SPARC FEL in original cascaded configurations.
\end{abstract}

\section{INTRODUCTION}

The SPARC experiment [13] is a unique facility to study seeded free electron lasers (FELs). The flexibility of the undulator together with the high brigthness electron beam will enable to test for the first time various techniques of frequency up conversion in a FEL.

It is now well known that seeded FELs [2, 3] offer several advantages with respect to SASE [1] FELs, such as temporal coherence and shot to shot stability. When seeding is combined with an undulator cascade, the properties of the fundamental can be transfered to the harmonics, and therefore allow delivery of high quality radiation at very short wavelength.

The SPARC FEL is being commissioned in the SASE mode around $500 \mathrm{~nm}$ [4]. The experimental setup for the generation of short wavelength seeds (from 400 to $114 \mathrm{~nm}$ ) is allready in place. Therefore, the seeding experiments at SPARC should soon start and provide results of interest for the community.

\section{THE SPARC FEL}

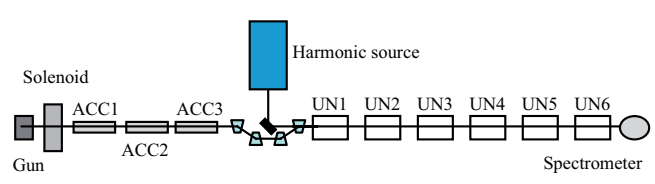

Figure 1: Experimental layout of the SPARC FEL.

\section{The LINAC}

The SPARC LINAC has been commissioned in 2008 [12]. The present layout is shown in Fig. 1. The injector is a SLAC/BNL/UCLA 1.6 cell S-band RF photoinjector operated with a UV laser pulse with flat top profile. At present time, the extracted charge is typically in the range of $300-500 \mathrm{pC}$. But further upgrade should allow to reach $1 \mathrm{nC}$ bunch charge. The beam is then accelerated up to $150 \mathrm{MeV}$ thanks to three SLAC type linac sections at $2.856 \mathrm{GHz}$. At the end of the last section, we expect an emittance of $\approx 1.2 \mathrm{~mm}$.mrad in both planes and an energy spread of $0.01 \%$. The emittance is measured by a quadrupole scan and the bunch length, slice emittance and slice energy spread by a high resolution RF deflector [14].

At the end of the LINAC, six quadrupoles on a drift section enable to match the electron beam at the entrance of the undulator. 


\section{The Undulator}

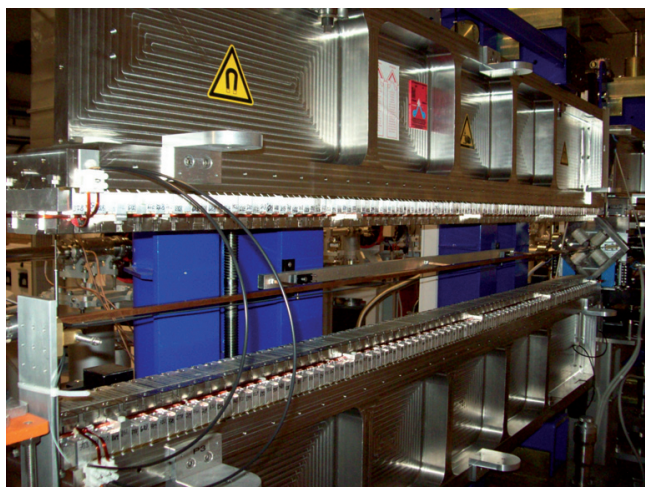

Figure 2: Picture of the SPARC undulator.

The SPARC undulator (see Fig. 2), realized by ACCEL Gmbh, consists of six planar sections. Each section is $2.156 \mathrm{~m}$ long: 77 periods of $28 \mathrm{~mm}$. Within the $50 \mathrm{~cm}$ drift space in between each section, is inserted a quadrupole to maintain the beam matching. Is also inserted a diagnostic chamber which allows to insert a YAG screen to visualize the electron beam position, or a mirror to extract the radiation and send it to a photodetector. Each section can be tuned independently to a gap varying from 8.2 to $20 \mathrm{~mm}$, i.e. to a deflexion parameter $K$ from 2.4 to 0.6. Therefore, the available resonance wavelength range on the fundamental is $\approx 200-600 \mathrm{~nm}$.

\section{EXPERIMENTAL SETUP FOR HARMONIC GENERATION}

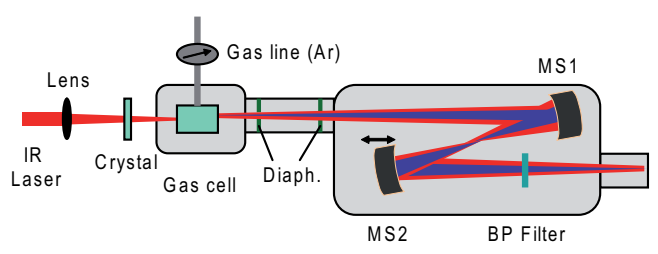

Figure 3: Experimental setup for harmonic generation in the SPARC hall. Depending on the ongoing experiment, the crystal or the gas cell is inserted on the IR laser path to perform harmonic generation. MS1 and MS2 are wo spherical mirrors. Diaph. are diaphragms to perform differential pumping. BP filter is a bandpass filter, centered on the harmonic wavelength under study.

The SPARC FEL is now being commissioned in the SASE mode. But its layout is particularly suited for a number of experiments where the FEL amplifier is seeded by an external laser source. A seed laser chain has been installed for this purpose. The seed laser is a Ti:Sa regenerative amplifier (LEGEND HFE by Coherent), driven by the same oscillator driving the photocathode amplifier. It delivers $2.5 \mathrm{~mJ}$ at $800 \mathrm{~nm}$ with a pulse duration shorter than $120 \mathrm{fs}$.
Harmonic generation is then performed to convert the infrared laser into UV. The experimental setup is presented in Fig. 3.

\section{Harmonic Generation in Crystal}

Second and third harmonic of the Ti:Sa laser source may be efficiently generated in an LBO crystal. This will allow to deliver UV pulses at 400 and $266 \mathrm{~nm}$ with an energy of a few tens of $\mu \mathrm{J}$.

\section{Harmonic Generation in Gas (HHG)}

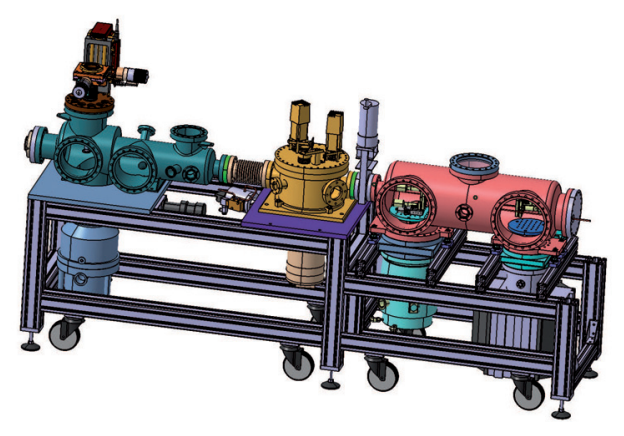

Figure 4: CATIA scheme of the harmonic generation chamber realized at CEA Saclay.

Shorter wavelengths will be obtained performing harmonic generation in rare gas (HHG) $[6,7]$. A dedicated chamber has been prepared at CEA-Saclay (France) and commissioned to SPARC in December 2007. A CATIA drawing of the chamber is given in Fig. 4. The infrared laser is focussed with a $2 \mathrm{~m}$ focussing lens and injected through a glass window into the chamber. At the focal point, the laser interacts the rare gas medium in a pulsed cell (cell filled up by bursts of gas at the laser repetition rate), to produce odd harmonics. This system will allow to deliver UV pulses at 266, 160 and $114 \mathrm{~nm}$ with an energy of respectively $1,0.1$ and $0.01 \mu \mathrm{J}$.

\section{EXPERIMENTAL SETUP FOR SEEDING}

The harmonic source, either obtained in crystal or in gas, is then refocussed using two spherical mirrors located inside the seeding chamber (see Fig. 2). Translating the second mirror allows to tune finally the focus point position within the first undulator section. The harmonic source is injected inside the undulator by means of a periscope and a magnetic chicane deflecting the electron beam from the straight path.

The undulator resonance wavelength is tuned at the seed wavelength by varying the beam energy in the range 155$200 \mathrm{MeV}$ and the undulator strength $K$ (via the gap).

Since both the seed and the electron beam result from the oscillator laser, they are naturally synchronised. The delay between the two pulses is visualized on a Streak Camera of 
2 ps-rms resolution and adjusted using an optical delay line located at the entrance of the regenerative amplifier.

The electron beam orbit in the undulator is given by the undulator magnetic axis. Once on this orbit, the electron beam position is first recorded on the YAG screens located in between the undulator sections. To ensure spatial overlap, the seed is then aligned with the periscope mirrors on the recorded positions.

\section{RADIATION DIAGNOSTICS}

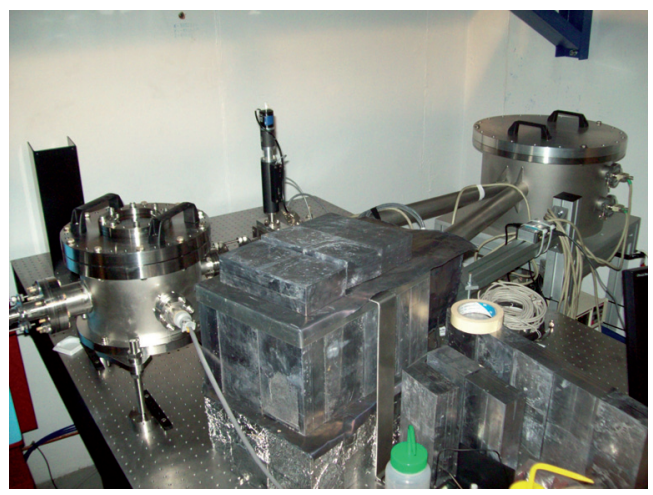

Figure 5: Undervacuum spectrometer installed at the end of the undulator.

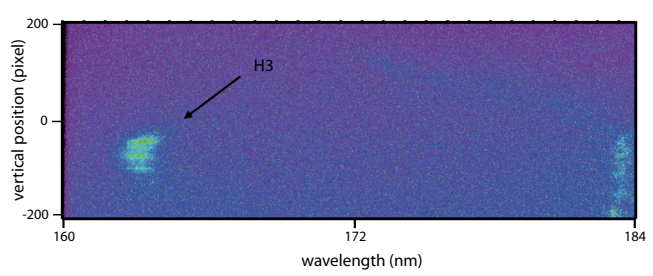

Figure 6: Image of the third harmonic of the SASE FEL recorded with the under vacuum spectrometer.

The SPARC experiment is equiped with several diagnostics. In between each undulator section, aluminium mirrors can extract the radiation and send it to in-air photodetectors (joulemeter, photodiode and spectrometer). In addition, an invacuum spectrometer built by the LUXOR laboratory (Padova, Italy) [10] is installed at the end of the undulator sequence. A picture is given in Fig. 5. The instrument consists of an entrance slit with variable width (up to $1 \mathrm{~mm}$ ) and a normal incidence grating imaging the slit on a Princeton UV grade CCD camera. The device enables the detection of spectra both in single shot and integrated mode in the spectral range of $40-570 \mathrm{~nm}$, together with the vertical distribution of the radiation. The observation of the third harmonic of the SASE FEL at $166 \mathrm{~nm}$ allowed to demonstrate the reliability of the measurement under vacuum (see Fig. 6). (a)
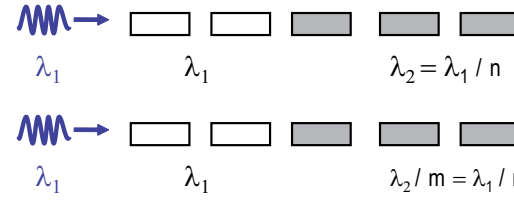

(b)

$$
\lambda_{2} / m=\lambda_{1} / n
$$

Figure 7: Undulator sections configuration for (a) single stage cascade FEL, (b) harmonic cascade FEL.

\section{FORESEEN SEEDING EXPERIMENTS}

\section{The FEL Amplifier}

The most simple seeding experiment consists in using the FEL as an amplifier of a low energy $(\approx n J)$ but short wavelength $(114 \mathrm{~nm})$ seed. We expect an amplification by more than three orders of magnitude on the fundamental, together with the generation of harmonic radiation $(55 \mathrm{~nm})$ with an energy close to $1 \mathrm{~nJ}$.

We also plan to verify the cleaning effect of the FEL on an HHG seed. Indeed, the typical structure of harmonics generated in gas consists in a sequence of attosecond pulses. According to simulations performed with PERSEO [16], the FEL slippage suppresses this structure along the undulator [19].

\section{The FEL Cascade}

The six sections of the SPARC undulator may be configured in order to set up a single stage cascaded FEL (see Fig. 7) based on a modulator-radiator configuration, similar to the one tested at BNL [17]. The number of sections of modulator and radiator may be adapted on the intensity of the available seed.

\section{The FEL Superradiant Cascade}

Using HHG seed pulses, the SPARC undulator will also allow to test for the first time the superradiant cascade concept [18] at $133 \mathrm{~nm}$. The feasibility of the experiment has been presented in [15].

\section{The FEL Harmonic Cascade}

Another original configuration will be tested: the FEL harmonic cascade [20]. In this case, the radiator is not tuned to an harmonic of the modulator: modulator and radiator have one harmonic in common, thus not of the same order. The configuration is illustrated in Fig. 7. At SPARC, according to simulations [15], we could for instance seed at $266 \mathrm{~nm}$ (with $1.5 \mu \mathrm{J}$ ), tune the modulator at $266 \mathrm{~nm}$ and the radiator at $200 \mathrm{~nm}$, and amplifiy at $66 \mathrm{~nm}$, which corresponds to the fourth harmonic of the modulator and the third harmonic of the radiator.

\section{CONCLUSION}

The SASE FEL is allready delivering radiation at $500 \mathrm{~nm}$ and its optimization is undergoing. The harmonic genera- 
tion setup is installed and has allready been tested. Therefore, the seeding experiments should start before the end of the year.

\section{ACKNOWLEDGMENTS}

This work was supported by the EU Commission in the Sixth Framework Program, Contract No. 011935EUROFEL.

\section{REFERENCES}

[1] R. Bonifacio et al., Opt. Comm. 50, 373 (1984)

[2] L.H. Yu, P.R.A. 44, 5178 (1991).

[3] L.H. Yu, Science. 289, 932 (2000).

[4] L. Giannessi et al., First Lasing at SPARC, these proceedings.

[5] L.H. Yu et al., P.R.L. 91, 074801 (2003).

[6] M. Ferray et al., J. Phys. B: At. Mol. Opt. Phys. 21, L31 (1988).

[7] A. McPherson et al., J. Opt. Soc. Am. B 4, 595 (1987).

[8] J.F. Hergott et al., Phys. Rev. A 66, 21801 (2002).

[9] L. Giannessi et al., www.sparc.it ref:SPARC-FEL-05/004.

[10] L. Poletto et al., Rev. Sci. Instr., 72 :2868, 2001. L. Poletto et al., Rev. Sci. Instr., 75 :4413, 2004.

[11] http://www-femtodrecam.cea.fr/slic/luca/luca1.htm.

[12] M. Ferrario et al., Proceedings of the FEL'08 Conference, Gyeongju, Korea.

[13] A. Renieri et al., Status report on SPARC project, in Proc. of the 26th FEL conference, Trieste 2004 JACoW (Joint Accelerator Conference Website) at http://www.JACoW.org, p. 163 (2004).

[14] A. Cianchi et al., Proceedings of the PAC'09 Conference, Vancouver, Canada (2009).

[15] L. Giannessi et al., Nucl. Instr. and Meth. A 593, 132 (2008).

[16] L. Giannessi, Overview of Perseo, a system for simulating FEL dynamics in Mathcad in Proc. of the 2006 FEL conference, JACoW (Joint Accelerator Con-ference Website) at http://www.JACoW.org, p. 91 (2006), see also http://www.perseo.enea.it.

[17] L. H. Yu et al., Phys. Rev. Lett. 91, 074801 (2003).

[18] L. Giannessi, P. Musumeci, S. Spampinati, J. Appl. Phys. 98, 043110 (2005).

[19] L. Giannessi et al., Proceedings of the FEL'06 Conference, Berlin, Germany, 95 (2006).

[20] L. Giannessi and P. Musumeci, New Journal of Physics 8, 294 (2006). 